\title{
Simultaneous Detection of 13 Allergens in Thermally Processed Food Using Targeted LC-MS/MS Approach
}

\author{
TAIRO OGURA \\ Shimadzu Corp., 1 Nishinokyo Kuwabara-cho, Nakagyo-ku, Kyoto 604-8511, Japan \\ Robert ClifFord \\ Shimadzu Scientific Instruments, Inc., 7102 Riverwood Dr, Columbia, MD 21044 \\ UWE OPPERMANN \\ Shimadzu Europa GmbH, Albert-Hahn-Straße 6-10, D-47269 Duisburg, Germany
}

\begin{abstract}
Food allergy is a major concern for public health and food industries. Because of the large numbers of food ingredients to be tested, MS is considered an alternative to existing techniques in terms of high selectivity, sensitivity, and capability to analyze multiple allergens simultaneously. In this study, we developed the method for monitoring significant peptides derived from 13 food allergens (milk, eggs, cod, shrimp, lobster, almonds, brazil nuts, cashew nuts, hazelnuts, walnuts, peanuts, wheat, and soybeans) and evaluated it in thermally processed foods (bread, cookie, fried fish, and frozen pasta). To select significant peptides to monitor, we used a bioinformatics-based approach and experimental confirmatory analysis. It was demonstrated that the developed method could detect target food ingredients from thermally processed foods successfully.
\end{abstract}

$\mathrm{F}$ ood allergy refers to an abnormal response of the immune system to certain food proteins $(1,2)$. Food allergy prevalence is currently estimated to be up to $10 \%$ of population in the developed world $(3,4)$. Because there is currently no cure for food allergies, strict avoidance of allergic substances is the only effective strategy for the management of a food allergy. The Food Allergen Labeling and Consumer Protection Act (FALCPA) requires that food manufacturers label food products intentionally containing eight major allergenic food ingredients (5). Milk, eggs, fish, crustacean shellfish, tree nuts, peanuts, wheat, and soybeans are identified as the major food allergens. On the other hand, the unexpected contamination of food by these allergens because of crosscontact during manufacturing or undeclared ingredients may cause the mislabeling of food. In the United States, 53 recalls caused by undeclared allergens were reported in 2017 , and the number of recalls increased at the rate of $15.9 \%$ per year on average from 2005 to 2017 (6).

Guest edited as a special report on "Mass Spectrometry: Status Quo in Food Allergen and Food Authenticity Applications" by Bert Popping and Carmen Diaz-Amigo.

Color images are available online at http://aoac.publisher.

ingentaconnect.com/content/aoac/jaoac

Corresponding author's e-mail: ogutairo@shimadzu.co.jp

DOI: https://doi.org/10.5740/jaoacint.19-0060
Immunological assays (e.g., ELISA and lateral flow assays) and PCR assays are the most commonly used routine techniques to detect allergenic foods. These techniques allow researchers to detect protein or DNA of allergenic substances with quick and easy sample handling; however, issues with these detection methods have also been reported, such as false positives caused by cross-reactivity and false detection of certain proteins in processed foods (7-9). These days, LC-tandem MS (MS/MS) is becoming an alternative technique for the detection of allergenic proteins with high selectivity and sensitivity as well as the capability of simultaneously analyzing multiple allergens. LC-MS/MS detects peptides as a significant trace of the proteins after sample preparation including extraction, reduction, alkylation, and enzymatic digestion, including the denaturation process in general. Unlike immunological assays, which rely on monoclonal or polyclonal antibody recognition of the epitope of target antigens, LC-MS/MS identifies denatured peptides by their chemical properties based on chromatography and MS (10).

Data-independent acquisition on high-resolution mass spectrometers has been demonstrated for the quantitative analysis of food allergens (11-13); however, targeted data acquisition using triple quadrupole mass spectrometers has been the most extensively used technique. To establish robust targeted data acquisition methods, the significant peptides need to be carefully selected based on specificity to the background matrix proteome and vulnerability after food processing (14).

In this study, we demonstrate the simultaneous analysis of multiple allergens of milk, eggs, cod, shrimp, lobster, almonds, brazil nuts, cashew nuts, hazelnuts, walnuts, peanuts, wheat, and soybeans and the detection of allergens in thermally processed foods such as bread, cookies, frozen pasta, and fried fish. The target foods were chosen based on FALCPA regulations. The selection of peptides for monitoring wheat glutenin was also discussed as an example of target peptides selection.

\section{Experimental}

\section{Protein Extraction, Reduction, Alkylation, Enzymatic Digestion, and Solid-Phase Extraction (SPE) Purification}

All chemicals and reagents were obtained from Sigma-Aldrich (St. Louis, MO) unless otherwise specified. Water and organic solvents were CHROMASOLVTM LC/MS grade, obtained from Honeywell (Morris Plains, NJ). Commercially available foods were obtained at local grocery stores. The samples were ground 
into fine powders with dry ice by a GM-200 knife mill (Retsch $\mathrm{GmbH}$, Haan, Germany). Ground samples (0.5 g each) were weighed into $15 \mathrm{~mL}$ centrifuge tubes. Five milliliters hexane was added into the tubes for defatting, and after centrifugation for $5 \mathrm{~min}$ at $5000 \times \mathrm{g}$, the hexane layer was discarded; the process was repeated a total of three times. Samples were dried using a nitrogen evaporator (MICROVAP; Organomation, Berlin, MA). The extraction buffer, containing $2 \mathrm{~mol} / \mathrm{L}$ urea, $1 \mathrm{mmol} / \mathrm{L}$ EDTA, $0.1 \mathrm{mmol} / \mathrm{L}$ phenylmethylsulfonyl fluoride, and $1 \mathrm{mmol} / \mathrm{L}$ dithiothreitol in $50 \mathrm{mmol} / \mathrm{L}$ Tris- $\mathrm{HCl}$ ( $\mathrm{pH}$ 8.0), was added for protein extraction. After incubation at room temperature for $3 \mathrm{~h}$ and centrifugation for $15 \mathrm{~min}$ at $5000 \times \mathrm{g}$, the supernatant was collected into a new $2 \mathrm{~mL}$ tube. Protein concentration was calculated using the Bicinchoninic Acid Protein Assay Kit. Aliquots of extracts containing $200 \mu \mathrm{g}$ proteins were transferred into a new $2 \mathrm{~mL}$ tube and were mixed with a reduction solution containing $8 \mathrm{~mol} / \mathrm{L}$ urea and $5 \mathrm{mmol} / \mathrm{L}$ dithiothreitol in $50 \mathrm{mmol} / \mathrm{L}$ Tris- $\mathrm{HCl}(\mathrm{pH} 8.0)$ for $60 \mathrm{~min}$. Proteins were mixed with $500 \mathrm{mmol} / \mathrm{L}$ iodoacetamide solution for $30 \mathrm{~min}$ in the dark for alkylation. For proteolysis with trypsin, the concentration of urea was diluted to $1 \mathrm{~mol} / \mathrm{L}$ with a digestion solution containing $50 \mathrm{mmol} / \mathrm{L}$ Tris- $\mathrm{HCl}(\mathrm{pH} \mathrm{8.0)}$ ), and MS grade Trypsin Gold (Promega, Madison, WI) was added into the tube at a ratio of 1:100 (total protein in sample:trypsin). Protein digestion was accomplished after incubation at $37^{\circ} \mathrm{C}$ for $12 \mathrm{~h}$. Ten microliters $50 \%$ formic acid in water was added to quench the reaction. After quenching the reaction, samples were cleaned up with an ISOLUTE ${ }^{\mathbb{R}}$ C18 SPE cartridge (25 mg/1 mL; Biotage, Uppsala, Sweden) and lyophilized with a FreeZone freeze dryer system (Labconco, Kansas City, MO). Dried samples were stored below $-20^{\circ} \mathrm{C}$ until analysis. Prior to analysis, dried samples were reconstituted with $50 \mu \mathrm{L} 1 \%$ formic acid and $2 \%$ acetonitrile in water and transferred into TORAST-H ${ }^{\text {TM }}$ Bio Vials (Shimadzu, Kyoto, Japan).

\section{Quantitation of Wheat Allergens}

Stable isotope-labeled synthetic peptides (heavy isotopelabeled peptides) were used for quantitation of native peptides (light peptides). Heavy-labeled peptides were labeled by incorporation of [13C615N2] lysine or [13C615N4] arginine located at each $\mathrm{C}$ terminus. Heavy-labeled peptides were purchased from Sigma-Aldrich. Lyophilized peptides were reconstituted to $50 \mathrm{pmol} / \mathrm{L}$ with $50 \%$ acetic acid in water as stock solutions. Lyophilized peptides and stock solutions were stored below $-80^{\circ} \mathrm{C}$ until use. Heavy-labeled peptides were spiked into each sample vial at the final concentration of $100 \mathrm{fmol} / \mu \mathrm{L}$. NIST wheat flour Standard Reference Material ${ }^{\circledR}$ (SRM) 1567B was used to prepare the calibration curve.

\section{LC-MS/MS Analysis}

LC-MS/MS analysis was conducted using a Nexera X2 Ultra HPLC system coupled to a triple quadrupole mass spectrometer (LCMS-8050; Shimadzu). For mobile phases, 0.1\% formic acid was used in water (A) and acetonitrile (B) at a flow rate of $0.5 \mathrm{~mL} / \mathrm{min}$. Ten microliters each sample was injected onto a Shim-pack XR-ODSIII analytical column $(2.0 \mathrm{~mm}$ id $\times 75 \mathrm{~mm}$ length, $1.6 \mu \mathrm{m})$ maintained at $40^{\circ} \mathrm{C}$. The gradient elution time program was set as follows: $2 \% \mathrm{~B}(0.0 \mathrm{~min}), 15 \% \mathrm{~B}$ (4.0 min), 40\% B (7.0 min), 95\% B (7.1-8.0 min), and 2\% B
(9.1-10.0 $\mathrm{min})$. For the rinse solvent, 70\% methanol in water was used. Samples were stored in an autosampler at $4^{\circ} \mathrm{C}$.

Peptides were monitored by single reaction monitoring, also known as multiple reaction monitoring, in positive electrospray ionization mode. The detection time window was set at $1 \mathrm{~min}$. The target cycle time was set at $1000 \mathrm{msec}$ with a $1 \mathrm{msec}$ pause between each transition. The dwell time for each transition was automatically set at 6-32 msec. Other parameters for the mass spectrometer were set as follows: interface voltage of $1 \mathrm{kV}$, nebulizing gas flow of $1.5 \mathrm{~L} / \mathrm{min}$, heating gas flow of $20 \mathrm{~L} / \mathrm{min}$, drying gas flow of $5 \mathrm{~L} / \mathrm{min}$, interface temperature of $250^{\circ} \mathrm{C}$, desolvation line temperature of $150^{\circ} \mathrm{C}$, heat block temperature of $200^{\circ} \mathrm{C}$, and collision-induced dissociation gas pressure of $270 \mathrm{kPa}$. All data were processed by LabSolutions (Shimadzu) and Skyline (15).

\section{Results and Discussion}

\section{Selection of Significant Peptides from Wheat Glutenin}

We monitored two subunits of glutenin as targets of wheat protein. Glutenin is the aggregate of high molecular weight glutenin subunit (HMW-GS) and low molecular weight glutenin subunit (LMW-GS). HMW-GS and LMW-GS account for 30 and $12 \%$ of storage protein in common wheat, respectively (16), and these are known as the major wheat allergens Tri a 26 and Tri a 36, respectively (17). First, four amino acid sequences for both glutenin subunits (P10385 and P10386 for Tri a 36, P10387 and P10388 for Tri a 26) were obtained from UniProt, and we prepared a method list for theoretically calculated tryptic peptides using Skyline. Among them, the only detectable peptides remaining in the list are potential target peptides. On the other hand, target peptides must be unique to background proteome matrices in order to avoid false positives. In the case of glutenin, the similar protein composites collectively referred to as gluten are found in other grains and grass families. We used a bioinformatics approach to select preferable peptides. The candidate peptides were gathered and used for a Basic Local Alignment Search Tool search using UniProt. The results were summarized in Tables 1 and 2. In Table 1, the results were merged into the genus. The "X" mark indicates that the peptide was found in the genus. The genus Triticum consists of many species of wheat, including common wheat ( $T$. aestivum). All of the peptides were found in Aegilops, including A. taushcii, which is the ancestor of wheat. Peptides that were frequently found among other grasses and grains, such as AQQLAAQLPAMCR, ELQESSLEACR, and EGGDALSASQ, were removed from the candidate list to avoid false positives. However, candidate peptides derived from common wheat may not be found in the other species in the genus Triticum. There are no peptides preserved in all species. This means that it is important to monitor multiple peptides to reduce the risk of false positives. ELQELQER, SVAVSQVAR, AQQPATQLPTVCR, VFLQQQCIPVAMQR, and VFLQQQCSPVAMPQR were finally selected as the target peptides. In addition to the bioinformatics approach, we analyzed eight grains, including common wheat and durum wheat, to evaluate these peptides experimentally (Figure 1).

Quantitation of food ingredients involves the normalization of multiple factors, such as extraction efficiency and the recovery of peptides during proteolysis. We used reference 
1318 Ogura et AL.: Journal of AOAC International Vol. 102, No. 5, 2019

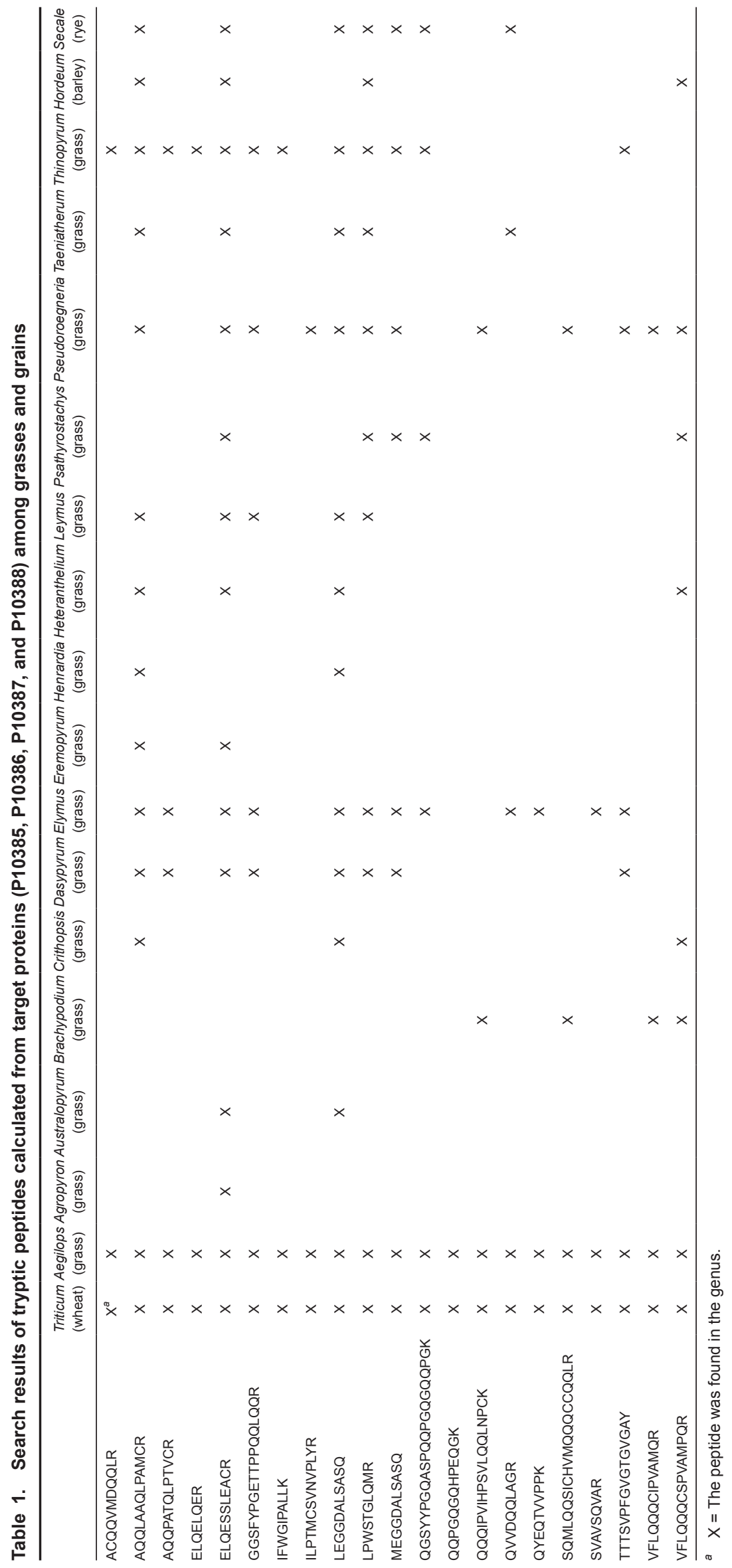




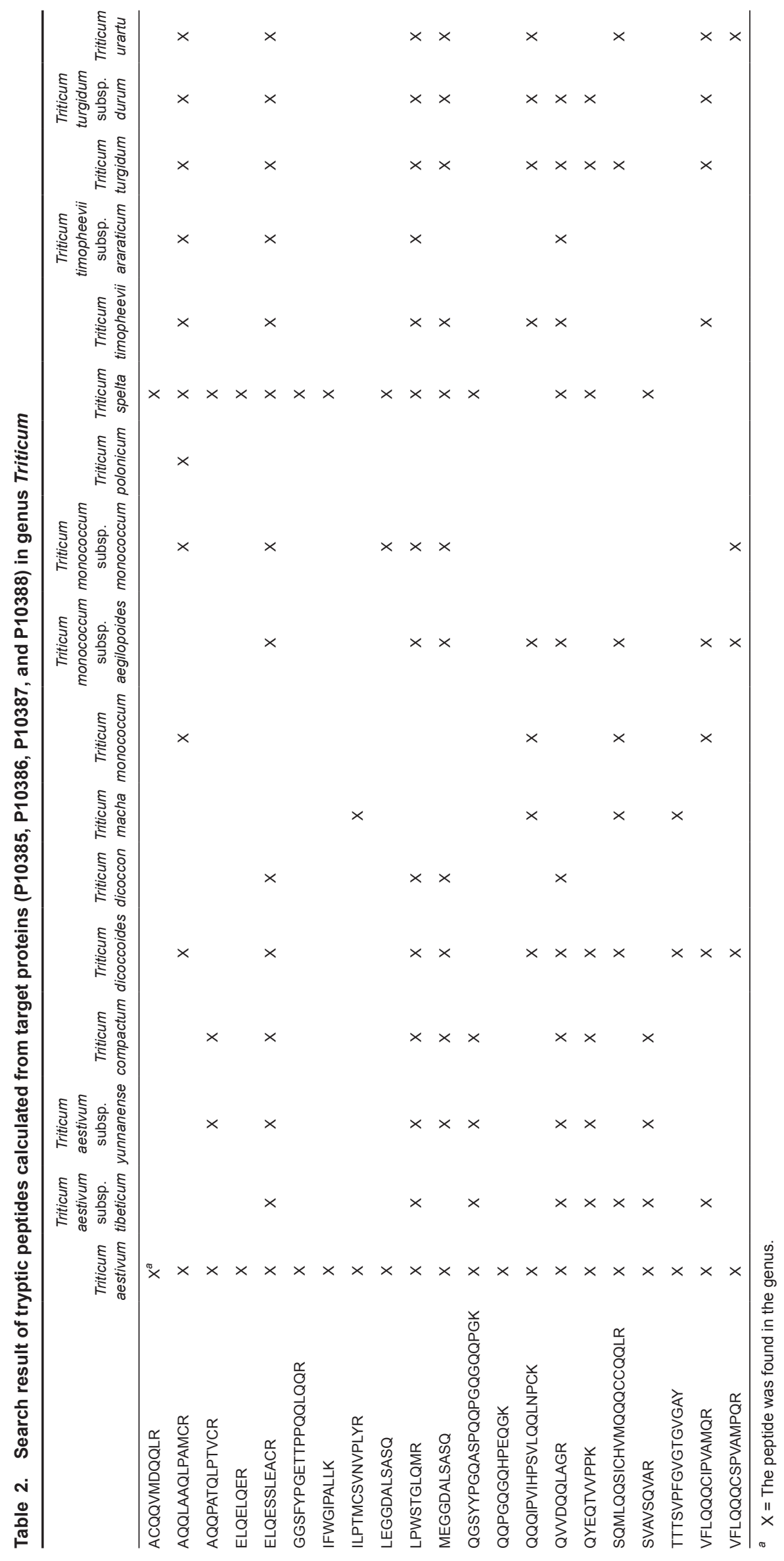


materials to assess the method's sensitivity. A calibration curve was prepared by plotting a peak ratio of light- and heavy-labeled peptides against the concentration of food ingredients. A 1/ concentration-weighted linear regression was used (Figure 2). The linear range was set at $3.9-250 \mathrm{ppm}(\mathrm{mg} / \mathrm{kg})$, and its $R^{2}$ value was $>0.995$.

\section{Detection of Multiple Allergens from Thermally Processed Foods}

We expanded the target of the method to detect tryptic peptides derived from 13 allergenic food ingredients: milk, eggs, cod, shrimp, lobster, almonds, brazil nuts, cashew nuts, hazelnuts, walnuts, peanuts, wheat, and soybeans. The final method contained 245 transitions of light peptides for simultaneous monitoring (Table 3). To evaluate the performance of this method, we analyzed a mixture of peptides prepared from raw food materials of milk, eggs, cod, shrimp, lobster, almonds, brazil nuts, cashew nuts, hazelnuts, walnuts, peanuts, wheat, and soybeans, and we confirmed that this method can detect all of the signals from the mixture of peptides prepared from raw materials (Figure 3 ). We also analyzed thermally processed foods such as bread, cookie, fried fish, and frozen pasta (Figure 4). These samples have labels declaring that they contain
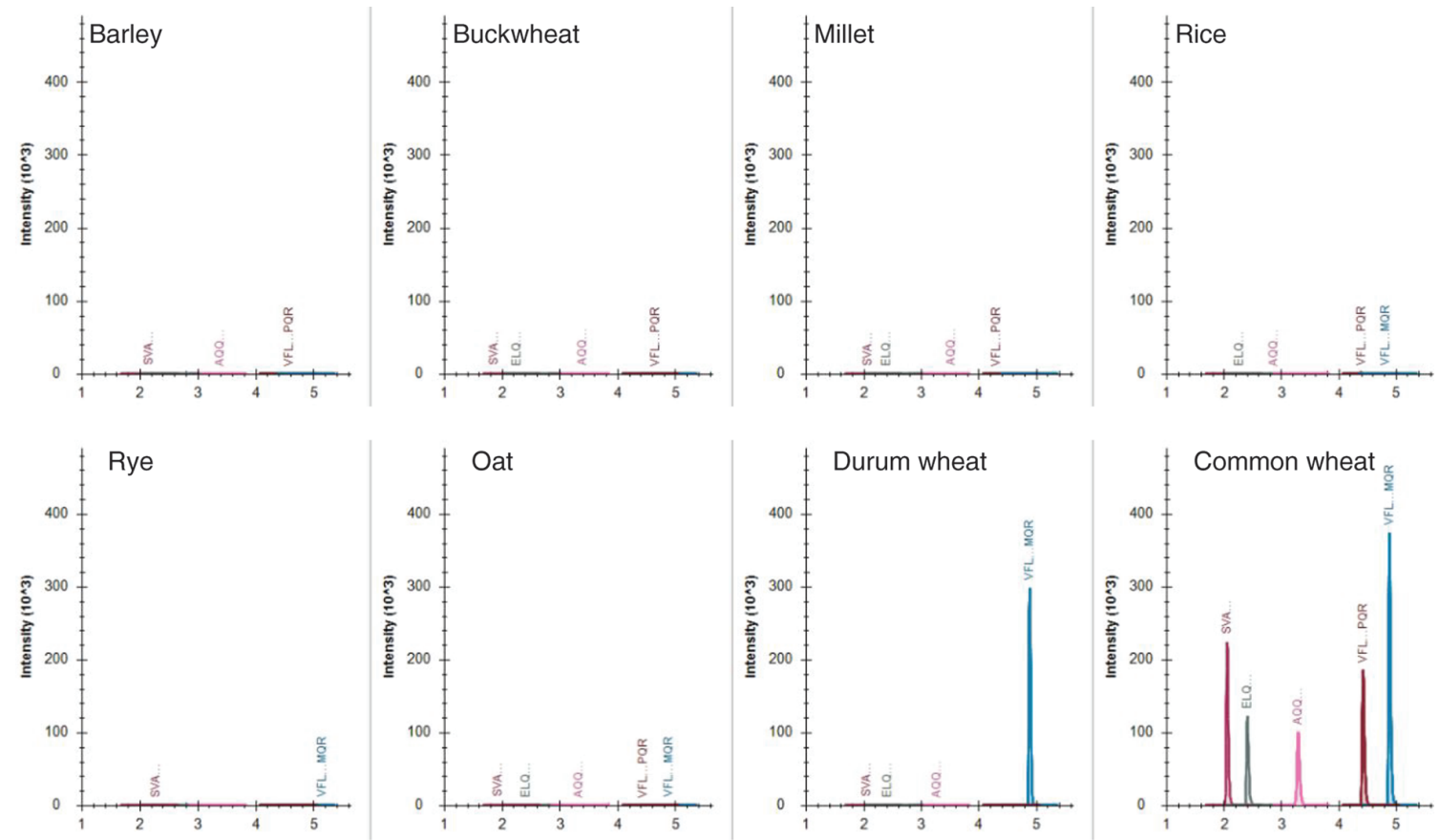

Figure 1. Chromatograms of five peptides derived from common wheat in barley, buckwheat, millet, rice, rye, oat, durum wheat, and common wheat.
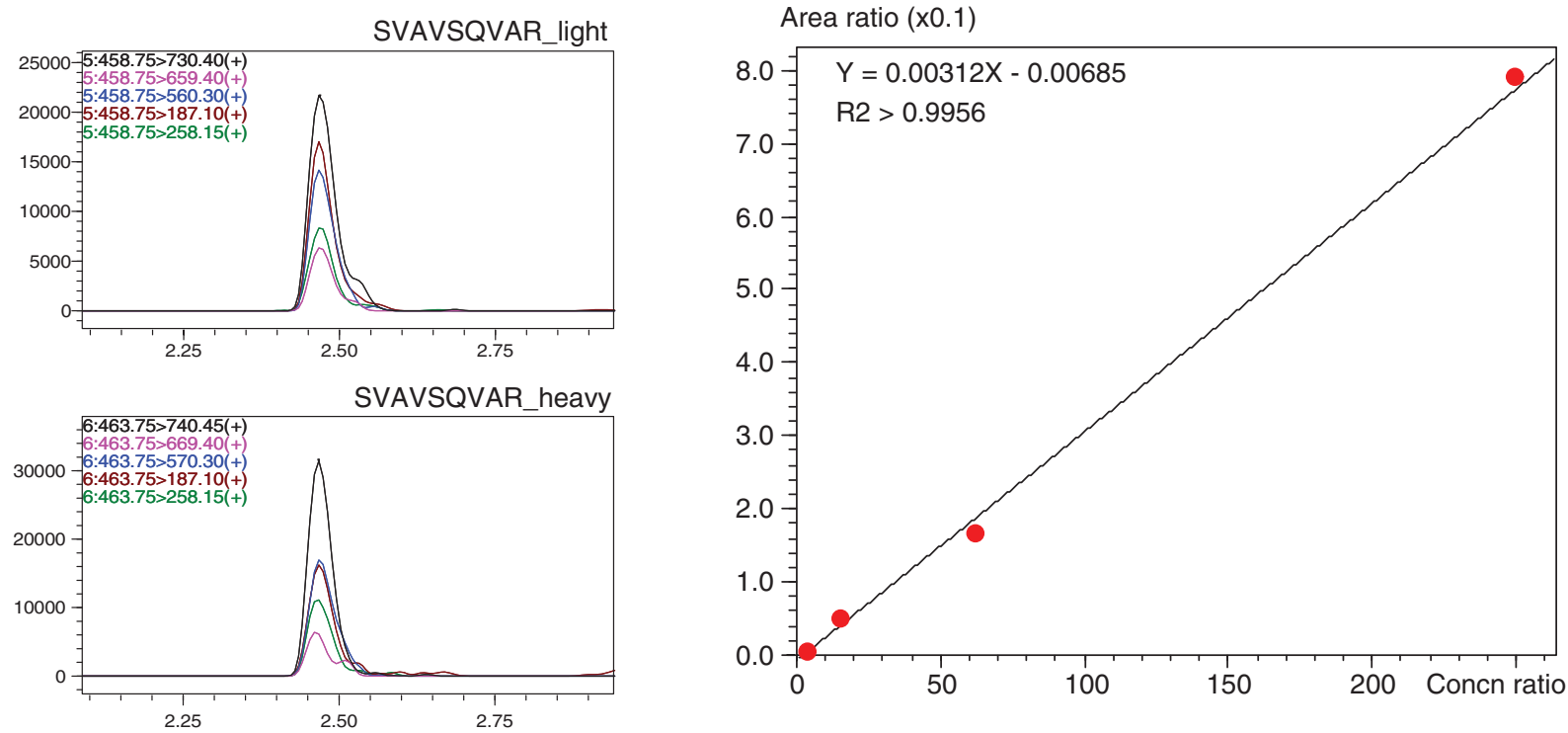

Figure 2. Representative chromatograms at $62.5 \mathrm{ppm}$ and calibration curve using NIST wheat flour SRM $1567 \mathrm{~B}$. 
Table 3. Transitions for target light peptides for milk, eggs, cod, shrimp, lobster, almonds, brazil nuts, cashew nuts, hazel nuts, walnuts, peanuts, wheat, and soybeans

\begin{tabular}{|c|c|c|c|c|c|c|}
\hline Food (binomial name) & Protein (IUIS ${ }^{a}$ name) & Compound name & $\begin{array}{l}\text { Precursor } \\
\quad \text { ion }\end{array}$ & Product ion & Collision energy, $\mathrm{V}$ & Ion name \\
\hline \multirow{25}{*}{$\begin{array}{l}\text { Common wheat } \\
\text { (Triticum aestivum) }\end{array}$} & \multirow{15}{*}{$\begin{array}{l}\text { High molecular } \\
\text { weight glutenin } \\
\quad \text { (Tri a 26) }\end{array}$} & \multirow[t]{5}{*}{ SVAVSQVAR } & \multirow[t]{5}{*}{458.75} & 730.40 & \multirow[t]{5}{*}{15.2} & y7 \\
\hline & & & & 659.40 & & y6 \\
\hline & & & & 560.30 & & y5 \\
\hline & & & & 187.10 & & b2 \\
\hline & & & & 258.15 & & b3 \\
\hline & & \multirow[t]{5}{*}{ AQQPATQLPTVCR } & \multirow[t]{4}{*}{735.40} & 1142.60 & \multirow[t]{4}{*}{23.8} & y10 \\
\hline & & & & 632.30 & & y5 \\
\hline & & & & 200.10 & & b2 \\
\hline & & & & 328.15 & & b3 \\
\hline & & & 490.60 & 632.30 & 12.9 & y5 \\
\hline & & \multirow[t]{5}{*}{ ELQELQER } & \multirow[t]{5}{*}{522.75} & 802.40 & \multirow[t]{5}{*}{17.2} & y6 \\
\hline & & & & 674.35 & & y5 \\
\hline & & & & 545.30 & & y4 \\
\hline & & & & 432.20 & & y3 \\
\hline & & & & 304.15 & & y2 \\
\hline & \multirow{10}{*}{$\begin{array}{l}\text { Low molecular } \\
\text { weight glutenin } \\
\text { GluB3-23 (Tri a 36) }\end{array}$} & \multirow[t]{5}{*}{ VFLQQQCIPVAMQR } & \multirow[t]{3}{*}{859.45} & 701.40 & \multirow[t]{3}{*}{27.6} & y6 \\
\hline & & & & 505.25 & & y4 \\
\hline & & & & 247.15 & & b2 \\
\hline & & & \multirow[t]{2}{*}{573.30} & 701.40 & \multirow[t]{2}{*}{15.8} & y6 \\
\hline & & & & 904.45 & & b7 \\
\hline & & \multirow[t]{5}{*}{ VFLQQQCSPVAMPQR } & 894.95 & 400.25 & 28.7 & y3 \\
\hline & & & \multirow[t]{4}{*}{596.95} & 798.45 & \multirow[t]{4}{*}{16.7} & $y 7$ \\
\hline & & & & 602.30 & & y5 \\
\hline & & & & 400.25 & & y3 \\
\hline & & & & 247.15 & & b2 \\
\hline \multirow[t]{20}{*}{ Milk (Bos taurus) } & \multirow[t]{20}{*}{ Caseins (Bos d 8) } & \multirow[t]{5}{*}{ FFVAPFPEVFGK } & 692.85 & 1090.60 & 22.5 & $y 10$ \\
\hline & & & & 991.50 & & y9 \\
\hline & & & & 920.50 & & y8 \\
\hline & & & & 676.35 & & y6 \\
\hline & & & & 351.20 & & y3 \\
\hline & & YLGYLEQLLR & 634.35 & 991.55 & 20.7 & y8 \\
\hline & & & & 771.45 & & y6 \\
\hline & & & & 658.40 & & y5 \\
\hline & & & & 277.15 & & b2 \\
\hline & & & & 334.20 & & b3 \\
\hline & & NAVPITPTLNR & 598.35 & 911.55 & 19.5 & y8 \\
\hline & & & & 701.40 & & y6 \\
\hline & & & & 600.35 & & y5 \\
\hline & & & & 186.10 & & b2 \\
\hline & & & & 285.15 & & b3 \\
\hline & & FALPQYLK & 490.30 & 832.50 & 16.2 & $y 7$ \\
\hline & & & & 761.45 & & y6 \\
\hline & & & & 648.35 & & y5 \\
\hline & & & & 423.25 & & y3 \\
\hline & & & & 147.10 & & $\mathrm{y} 1$ \\
\hline
\end{tabular}


1322 Ogura et AL.: Journal of AOAC International Vol. 102, No. 5, 2019

Table 3. (continued)

\begin{tabular}{|c|c|c|c|c|c|c|}
\hline Food (binomial name) & Protein (IUIS ${ }^{a}$ name) & Compound name & $\begin{array}{l}\text { Precursor } \\
\text { ion }\end{array}$ & Product ion & Collision energy, $\mathrm{V}$ & Ion name \\
\hline & Beta-lactoglobulin & IDALNENK & 458.75 & 803.40 & 15.2 & y7 \\
\hline & & & & 688.35 & & y6 \\
\hline & & & & 504.25 & & y4 \\
\hline & & & & 229.10 & & b2 \\
\hline & & & & 300.15 & & b3 \\
\hline \multirow[t]{15}{*}{ Egg (Gallus gallus) } & Ovalbumin (Gal d 2) & NVLQPSSVDSQTAMVLVNAIVFK & 820.80 & 214.10 & 24.7 & b2 \\
\hline & & & & 327.20 & & b3 \\
\hline & & & & 455.25 & & b4 \\
\hline & & & & 903.55 & & y8 \\
\hline & & & & 790.50 & & y7 \\
\hline & Ovotransferrin & ATYLDCIK & 492.25 & 811.40 & 16.3 & y6 \\
\hline & (Gal d 3) & & & 648.35 & & y5 \\
\hline & & & & 535.25 & & y4 \\
\hline & & & & 420.25 & & y3 \\
\hline & & & & 173.10 & & b2 \\
\hline & & TDERPASYFAVAVAR & 551.60 & 586.35 & 15.1 & y6 \\
\hline & & & & 515.35 & & y5 \\
\hline & & & & 416.25 & & y4 \\
\hline & & & & 345.20 & & y3 \\
\hline & & & & 920.40 & & b8 \\
\hline \multirow{15}{*}{$\begin{array}{l}\text { Atlantic cod (Gadus } \\
\text { morhua) }\end{array}$} & Beta-parvalbumin & ALTDAETK & 424.70 & 664.30 & 14.2 & y6 \\
\hline & (Gad m 1) & & & 563.25 & & y5 \\
\hline & & & & 448.25 & & y4 \\
\hline & & & & 248.15 & & y2 \\
\hline & & & & 185.15 & & b2 \\
\hline & & AFFVIDQDK & 541.80 & 864.45 & 17.8 & $y 7$ \\
\hline & & & & 717.40 & & y6 \\
\hline & & & & 618.30 & & y5 \\
\hline & & & & 219.10 & & b2 \\
\hline & & & & 366.20 & & b3 \\
\hline & & SGFIEEDELK & 583.80 & 875.45 & 19.1 & $y 7$ \\
\hline & & & & 762.35 & & y6 \\
\hline & & & & 633.30 & & y5 \\
\hline & & & & 504.25 & & y4 \\
\hline & & & & 260.20 & & y2 \\
\hline \multirow{10}{*}{$\begin{array}{l}\text { Whiteleg shrimp } \\
\text { (Litopenaeus } \\
\text { vannamei) }\end{array}$} & Tropomyosin (Lit $\vee 1)$ & IQLLEEDLER & 629.35 & 242.15 & 20.5 & b2 \\
\hline & & & & 355.25 & & b3 \\
\hline & & & & 1016.55 & & y8 \\
\hline & & & & 903.45 & & $y 7$ \\
\hline & & & & 790.35 & & y6 \\
\hline & & IVELEEELR & 565.30 & 213.15 & 18.5 & b2 \\
\hline & & & & 342.20 & & b3 \\
\hline & & & & 917.45 & & $y 7$ \\
\hline & & & & 788.40 & & y6 \\
\hline & & & & 675.35 & & y5 \\
\hline
\end{tabular}


Table 3. (continued)

\begin{tabular}{|c|c|c|c|c|c|c|}
\hline Food (binomial name) & Protein (IUIS ${ }^{a}$ name) & Compound name & $\begin{array}{l}\text { Precursor } \\
\text { ion }\end{array}$ & Product ion & Collision energy, $\mathrm{V}$ & Ion name \\
\hline & \multirow{10}{*}{$\begin{array}{l}\text { Sarcoplasmic } \\
\text { calcium-binding } \\
\text { protein (Lit } \vee 4)\end{array}$} & \multirow[t]{5}{*}{ VFIANQFK } & \multirow[t]{5}{*}{483.75} & 247.15 & \multirow[t]{5}{*}{16} & b2 \\
\hline & & & & 867.45 & & $y 7$ \\
\hline & & & & 720.40 & & y6 \\
\hline & & & & 607.30 & & y5 \\
\hline & & & & 536.30 & & y4 \\
\hline & & \multirow[t]{5}{*}{ AGGLTLER } & \multirow[t]{5}{*}{408.75} & 129.05 & \multirow[t]{5}{*}{13.7} & b2 \\
\hline & & & & 745.40 & & $y 7$ \\
\hline & & & & 688.40 & & y6 \\
\hline & & & & 518.30 & & y4 \\
\hline & & & & 417.25 & & y3 \\
\hline & \multirow{10}{*}{$\begin{array}{l}\text { Myosin, light chain } 2 \\
\quad(\text { Lit } \vee 3)\end{array}$} & \multirow[t]{5}{*}{ EGFQLMDR } & \multirow[t]{5}{*}{498.25} & 662.35 & \multirow[t]{5}{*}{16.4} & y5 \\
\hline & & & & 534.25 & & y4 \\
\hline & & & & 421.20 & & y3 \\
\hline & & & & 290.15 & & y2 \\
\hline & & & & 175.10 & & $\mathrm{y} 1$ \\
\hline & & \multirow[t]{5}{*}{ GTFDEIGR } & \multirow[t]{5}{*}{447.70} & 736.35 & \multirow[t]{5}{*}{14.9} & y6 \\
\hline & & & & 589.30 & & y5 \\
\hline & & & & 474.25 & & y4 \\
\hline & & & & 345.20 & & y3 \\
\hline & & & & 232.15 & & y2 \\
\hline \multirow{15}{*}{$\begin{array}{l}\text { American lobster } \\
\text { (Homarus americanus) }\end{array}$} & \multirow{15}{*}{$\begin{array}{c}\text { Tropomyosin } \\
\text { (Hom a 1) }\end{array}$} & \multirow[t]{5}{*}{ ALQNAEGEVAALNR } & \multirow[t]{5}{*}{728.40} & 1029.55 & \multirow[t]{5}{*}{23.6} & $y 10$ \\
\hline & & & & 958.50 & & y9 \\
\hline & & & & 829.45 & & y8 \\
\hline & & & & 544.30 & & y5 \\
\hline & & & & 636.30 & & $\mathrm{y} 12$ \\
\hline & & \multirow[t]{5}{*}{ FLAEEADR } & \multirow[t]{5}{*}{475.75} & 690.30 & \multirow[t]{5}{*}{15.7} & y6 \\
\hline & & & & 619.25 & & y5 \\
\hline & & & & 490.25 & & y4 \\
\hline & & & & 175.10 & & $\mathrm{y} 1$ \\
\hline & & & & 261.15 & & b2 \\
\hline & & SITDELDQTFSELSGY & 902.90 & 439.20 & 29 & y4 \\
\hline & & & & 326.15 & & y3 \\
\hline & & & & 239.10 & & y2 \\
\hline & & & & 182.10 & & $\mathrm{y} 1$ \\
\hline & & & & 120.05 & & y2 \\
\hline Soybean (Glycine max) & Trypsin inhibitor & NKPLVVEFQK & 601.35 & 243.15 & 19.6 & b2 \\
\hline & (Gly m TI) & & & 959.55 & & y8 \\
\hline & & & 401.25 & 453.30 & 9.6 & b4 \\
\hline & & & & 552.35 & & b5 \\
\hline & & & & 422.25 & & y3 \\
\hline & & NKPLVVQFQK & 600.85 & 958.55 & 19.6 & y8 \\
\hline & & & 400.90 & 453.30 & 9.6 & b4 \\
\hline & & & & 552.35 & & b5 \\
\hline & & & & 649.35 & & y5 \\
\hline & & & & 550.30 & & $\mathrm{y} 4$ \\
\hline & & CPLTVVQSR & 530.30 & 371.15 & 17.4 & b3 \\
\hline & & & & 802.50 & & $y 7$ \\
\hline
\end{tabular}


1324 Ogura et AL.: Journal of AOAC International Vol. 102, No. 5, 2019

Table 3. (continued)

\begin{tabular}{|c|c|c|c|c|c|c|}
\hline Food (binomial name) & Protein (IUIS ${ }^{a}$ name) & Compound name & $\begin{array}{l}\text { Precursor } \\
\text { ion }\end{array}$ & Product ion & Collision energy, $\mathrm{V}$ & Ion name \\
\hline & & & & 689.40 & & y6 \\
\hline & & & & 588.35 & & y5 \\
\hline & & & & 489.30 & & y4 \\
\hline & & GIGTIISSPYR & 582.30 & 993.55 & 19.1 & y9 \\
\hline & & & & 835.45 & & $y 7$ \\
\hline & & & & 722.40 & & y6 \\
\hline & & & & 609.30 & & y5 \\
\hline & & & & 522.25 & & y4 \\
\hline \multirow{6}{*}{$\begin{array}{l}\text { Peanut (Arachis } \\
\text { hypogaea) }\end{array}$} & \multirow{6}{*}{$\begin{array}{l}\text { Cupin vicillin-type, } \\
\text { 7S globulin (Ara h 1) }\end{array}$} & NNPFYFPSR & 571.25 & 229.10 & 18.7 & b2 \\
\hline & & & & 669.35 & & y5 \\
\hline & & & & 506.25 & & y4 \\
\hline & & GTGNLELVAVR & 564.80 & 686.40 & 18.5 & y6 \\
\hline & & & & 557.40 & & y5 \\
\hline & & & & 444.30 & & y4 \\
\hline \multirow{20}{*}{$\begin{array}{l}\text { Brazil nut (Bertholletia } \\
\quad \text { excelsa) }\end{array}$} & \multirow{20}{*}{$\begin{array}{l}\text { 11S globulin } \\
\text { (Ber e 2) }\end{array}$} & NTIRPQGLLLPVYTNAPK & 665.70 & 889.50 & 19.2 & y8 \\
\hline & & & & 890.55 & & $\mathrm{y} 16$ \\
\hline & & & & 445.25 & & y8 \\
\hline & & & & 880.50 & & b8 \\
\hline & & & & 440.75 & & b8 \\
\hline & & LYYVTQGR & 500.25 & 723.40 & 16.5 & y6 \\
\hline & & & & 560.30 & & y5 \\
\hline & & & & 461.25 & & y4 \\
\hline & & & & 277.15 & & b2 \\
\hline & & & & 440.20 & & b3 \\
\hline & & LNANSVVYAVR & 603.35 & 978.55 & 19.7 & y9 \\
\hline & & & & 907.50 & & y8 \\
\hline & & & & 607.35 & & y5 \\
\hline & & & & 228.15 & & b2 \\
\hline & & & & 299.15 & & b3 \\
\hline & & GIPVGVLANAYR & 615.35 & 962.55 & 20.1 & y9 \\
\hline & & & & 863.45 & & y8 \\
\hline & & & & 707.40 & & y6 \\
\hline & & & & 530.30 & & y10 \\
\hline & & & & 707.45 & & b8 \\
\hline \multirow{14}{*}{$\begin{array}{l}\text { Cashew (Anacardium } \\
\text { occidentale) }\end{array}$} & \multirow{14}{*}{$\begin{array}{l}\text { 11S seed storage } \\
\text { protein (Ana o 2) }\end{array}$} & NLFSGFDTELLAEAFQVDER & 767.70 & 993.45 & 22.8 & y8 \\
\hline & & & & 793.40 & & y6 \\
\hline & & & & 646.30 & & y5 \\
\hline & & & & 304.15 & & y2 \\
\hline & & ADIYTPEVGR & 560.80 & 821.40 & 18.4 & $y 7$ \\
\hline & & & & 658.35 & & y6 \\
\hline & & & & 557.30 & & y5 \\
\hline & & & & 300.15 & & b3 \\
\hline & & & & 564.25 & & b5 \\
\hline & & AMTSPLAGR & 452.25 & 701.40 & 15 & $y 7$ \\
\hline & & & & 600.35 & & y6 \\
\hline & & & & 513.30 & & y5 \\
\hline & & & & 175.10 & & $\mathrm{y} 1$ \\
\hline & & & & 203.10 & & b2 \\
\hline
\end{tabular}


Table 3. (continued)

\begin{tabular}{|c|c|c|c|c|c|c|}
\hline Food (binomial name) & Protein (IUIS ${ }^{a}$ name) & Compound name & $\begin{array}{l}\text { Precursor } \\
\text { ion }\end{array}$ & Product ion & Collision energy, $\mathrm{V}$ & Ion name \\
\hline & & TSVLGGMPEEVLANAFQISR & 707.05 & 906.50 & 20.7 & y8 \\
\hline & & & & 835.45 & & y7 \\
\hline & & & & 650.35 & & y5 \\
\hline & & & & 503.30 & & y4 \\
\hline & & & & 262.15 & & y2 \\
\hline \multirow{10}{*}{$\begin{array}{l}\text { English walnut } \\
\text { (Juglans regia) }\end{array}$} & \multirow{10}{*}{$\begin{array}{l}\text { Seed storage protein } \\
\text { (Jug } \mathrm{r} 4)\end{array}$} & TIEPNGLLLPQYSNAPQLVYIAR & 857.45 & 1144.65 & 26.1 & y10 \\
\hline & & & & 959.55 & & y8 \\
\hline & & & & 522.30 & & y4 \\
\hline & & & & 810.45 & & y14 \\
\hline & & & & 480.30 & & y8 \\
\hline & & NEGFEWVSFK & 621.80 & 999.50 & 20.3 & y8 \\
\hline & & & & 942.45 & & y7 \\
\hline & & & & 795.40 & & y6 \\
\hline & & & & 381.20 & & y3 \\
\hline & & & & 244.10 & & b2 \\
\hline \multirow{10}{*}{$\begin{array}{l}\text { Black walnut } \\
\text { (Juglans nigra) }\end{array}$} & \multirow[t]{10}{*}{ Legumin (Jug n 4) } & LVALEPSNR & 499.80 & 786.40 & 16.5 & y7 \\
\hline & & & & 715.35 & & y6 \\
\hline & & & & 602.30 & & y5 \\
\hline & & & & 473.25 & & y4 \\
\hline & & & & 213.15 & & b2 \\
\hline & & GITGVLFPGCPETFEESQQGQSR & 842.05 & 919.40 & 25.5 & y8 \\
\hline & & & & 790.40 & & $y 7$ \\
\hline & & & & 447.25 & & y4 \\
\hline & & & & 992.45 & & y17 \\
\hline & & & & 918.90 & & $\mathrm{y} 16$ \\
\hline \multirow{20}{*}{$\begin{array}{l}\text { European hazel } \\
\text { (Corylus avellana) }\end{array}$} & \multirow{10}{*}{$\begin{array}{c}48 \text { kDa glycoprotein } \\
\text { (Cor a 11) }\end{array}$} & GNIVNEFER & 539.25 & 793.40 & 17.7 & y6 \\
\hline & & & & 580.25 & & y4 \\
\hline & & & & 226.10 & & y3 \\
\hline & & & & 172.05 & & b2 \\
\hline & & & & 285.15 & & b3 \\
\hline & & ELAFNLPSR & 523.80 & 804.45 & 17.2 & y7 \\
\hline & & & & 733.40 & & y6 \\
\hline & & & & 586.35 & & y5 \\
\hline & & & & 472.30 & & y4 \\
\hline & & & & 359.20 & & y3 \\
\hline & \multirow{10}{*}{$\begin{array}{l}\text { 11S globulin-like } \\
\text { protein (Cor a 9) }\end{array}$} & \multirow[t]{5}{*}{ ADIYTEQVGR } & 576.30 & 852.40 & 18.9 & $y 7$ \\
\hline & & & & 689.35 & & y6 \\
\hline & & & & 588.30 & & y5 \\
\hline & & & & 459.25 & & y4 \\
\hline & & & & 300.15 & & b3 \\
\hline & & \multirow[t]{5}{*}{ INTVNSNTLPVLR } & 720.90 & 1013.55 & 23.3 & y9 \\
\hline & & & & 484.30 & & y4 \\
\hline & & & & 228.15 & & b2 \\
\hline & & & & 329.20 & & b3 \\
\hline & & & 480.95 & 484.30 & 12.5 & y4 \\
\hline
\end{tabular}


Table 3. (continued)

\begin{tabular}{|c|c|c|c|c|c|c|}
\hline Food (binomial name) & Protein (IUIS ${ }^{a}$ name) & Compound name & $\begin{array}{l}\text { Precursor } \\
\text { ion }\end{array}$ & Product ion & Collision energy, $\mathrm{V}$ & Ion name \\
\hline & & ALPDDVLANAFQISR & 815.45 & 1019.55 & 26.3 & y9 \\
\hline & & & & 906.50 & & y8 \\
\hline & & & & 835.45 & & $y 7$ \\
\hline & & & & 723.35 & & $y 13$ \\
\hline & & & 543.95 & 723.35 & 14.8 & $\mathrm{y} 13$ \\
\hline \multirow[t]{10}{*}{ Almond (Prunus dulcis) } & Prunin 1 (Pru du 6) & ALPDEVLANAYQISR & 830.45 & 738.40 & 26.7 & b7 \\
\hline & & & & 1035.55 & & y9 \\
\hline & & & & 922.45 & & y8 \\
\hline & & & 553.95 & 738.40 & 15.1 & b7 \\
\hline & & & & 503.30 & & $\mathrm{y} 4$ \\
\hline & Prunin 2 (Pru du 6) & ALPDEVLQNAFR & 686.85 & 185.15 & 22.3 & b2 \\
\hline & & & & 1188.60 & & $y 10$ \\
\hline & & & & 748.40 & & y6 \\
\hline & & & & 635.35 & & y5 \\
\hline & & & & 507.25 & & y4 \\
\hline
\end{tabular}

a IUIS = International Union of Immunological Societies.

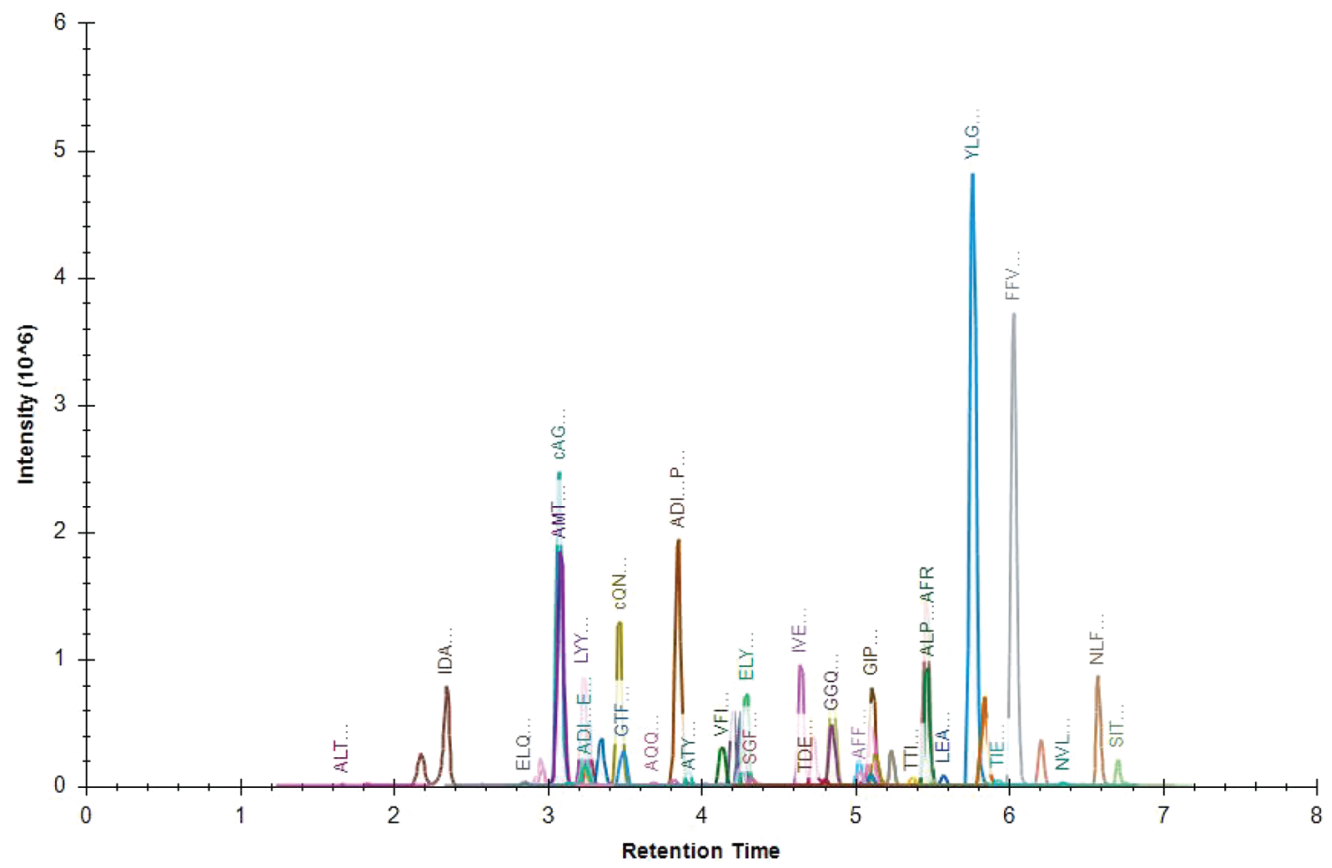

Figure 3. Chromatogram of a mixture of milk, eggs, cod, shrimp, lobster, almonds, brazil nuts, cashew nuts, hazelnuts, walnuts, peanuts, wheat, and soybeans.

or can reasonably be expected to contain wheat, milk, eggs, peanuts, soybeans, cod, or shrimp as allergenic food ingredients (Table 4). A comparison of chromatograms of frozen pasta and standard samples is shown in Figure 5. The ratio of wheat-related peptides in frozen pasta suggested the existence of both common wheat and durum wheat, and a majority was durum wheat, which is commonly used to produce dry pasta (Figure $5 \mathrm{c}$ and $5 \mathrm{~d}$ ). In contrast, soybean peptides were not detected in frozen pasta despite their declaration (Figure 5e and 5f). The manufacturer listed soybean oil as an ingredient on the product label of the frozen pasta, although it is known that highly purified oil does not contain protein. It suggested that the food samples did not contain soybean proteins.

\section{Conclusions}

We developed the LC-MS/MS method for simultaneous monitoring of 13 allergenic food ingredients, including at least one food ingredient from the eight foods and food groups required by FALCPA. A total of 245 transitions were 
a) Bread

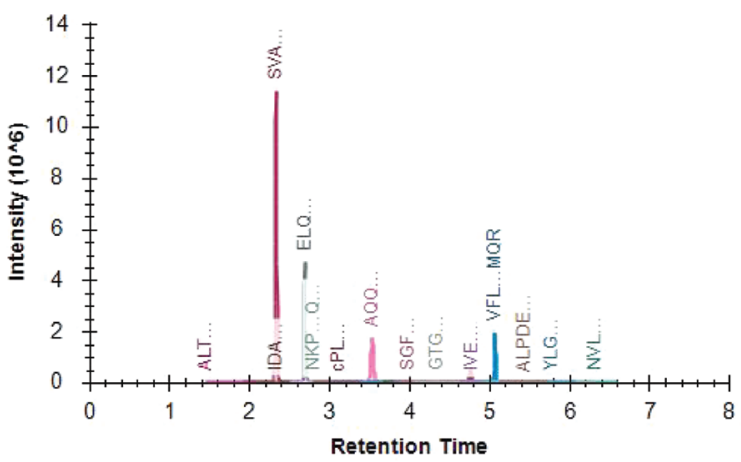

c) Fried fish "beer batter cod"

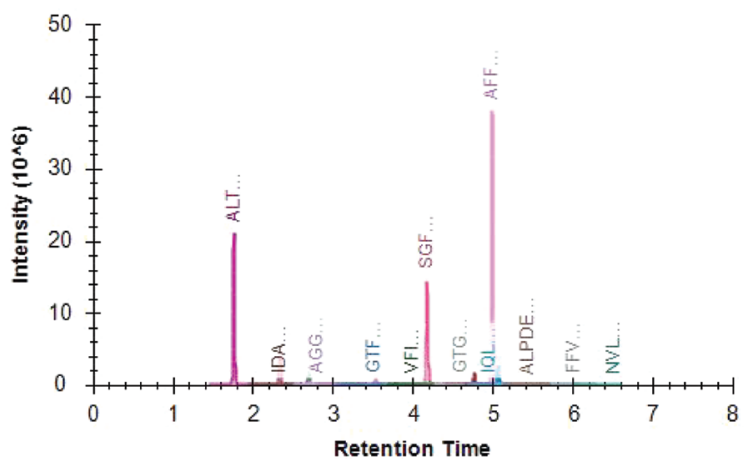

b) Cookies

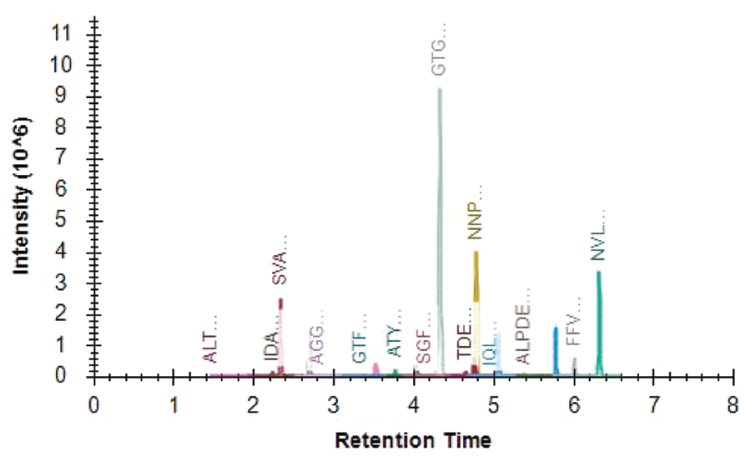

d) Frozen pasta "qarlic shrimp"

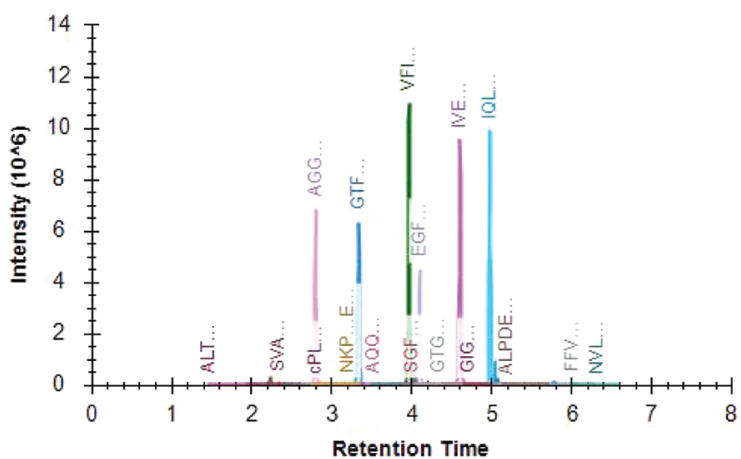

Figure 4. Chromatograms of four thermally processed foods: (a) bread, (b) cookies, (c) fried fish, and (d) frozen pasta.

Table 4. Summary of food labeling and analytical results of thermally processed foods (bread, peanuts cookie, fried fish, and frozen pasta)

\begin{tabular}{|c|c|c|c|c|c|c|c|c|c|}
\hline \multirow[b]{2}{*}{ Food } & \multirow[b]{2}{*}{ Allergens } & \multicolumn{2}{|c|}{ Bread } & \multicolumn{2}{|c|}{ Cookie $^{a}$} & \multicolumn{2}{|c|}{$\begin{array}{l}\text { Fried fish, "beer } \\
\text { butter cod" }\end{array}$} & \multicolumn{2}{|c|}{$\begin{array}{l}\text { Frozen pasta, } \\
\text { "garlic shrimp" }\end{array}$} \\
\hline & & Label & Detect & Label & Detect & Label & Detect & Label & Detect \\
\hline \multirow[t]{2}{*}{ Wheat } & High molecular weight glutenin (Tri a 26) & $x^{b}$ & $\mathrm{x}$ & $-^{c}$ & $\mathrm{x}$ & $x$ & $\mathrm{x}$ & $\mathrm{x}$ & $x$ \\
\hline & Low molecular weight glutenin (Tri a 36) & & $x$ & & $x$ & & $x$ & & $x$ \\
\hline \multirow[t]{2}{*}{ Milk } & Caseins (Bos d 8) & & & - & $\mathrm{x}$ & $\mathrm{x}$ & $x$ & $\mathrm{x}$ & $x$ \\
\hline & Beta-lactoglobulin (Bos d 5) & & & & $x$ & & $x$ & & $x$ \\
\hline \multirow[t]{2}{*}{ Eggs } & Ovalbumin (Gal d 2) & $\mathrm{x}$ & $\mathrm{x}$ & - & $\mathrm{x}$ & & & & \\
\hline & Ovotransferrin (Gal d 3) & & $x$ & & $x$ & & & & \\
\hline Peanuts & Cupin, vicillin-type, 7S globulin (Ara h 1) & & & - & $x$ & & & & \\
\hline Soybeans & Trypsin inhibitor (Gly m TI) & & & - & & & & $\mathrm{x}$ & \\
\hline Atlantic cod & Beta-parvalbumin (Gad m 1) & & & - & & $x$ & $x$ & & \\
\hline \multirow[t]{3}{*}{ Whiteleg shrimp } & Tropomyosin (Lit v 1) & & & - & & & & $x^{d}$ & $x$ \\
\hline & Myosin, light chain 2 (Lit v 3 ) & & & & & & & & $x$ \\
\hline & Sarcoplasmic CBP (Lit v 4) & & & & & & & & $x$ \\
\hline
\end{tabular}

\footnotetext{
a No allergen information was provided.

$b \quad \mathrm{x}=$ Declared on label and/or detected from samples.

c $-=$ Not applicable.

d It was declared as shrimp.
} 
a) Shrimp peptides in frozen pasta

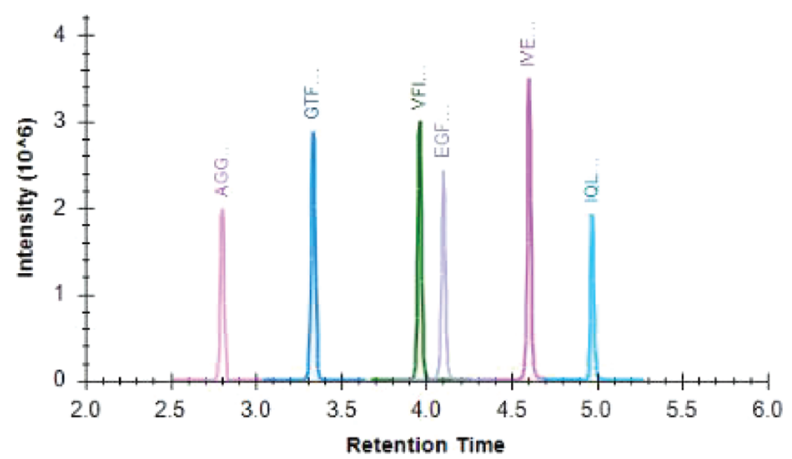

c) Wheat peptides in frozen pasta

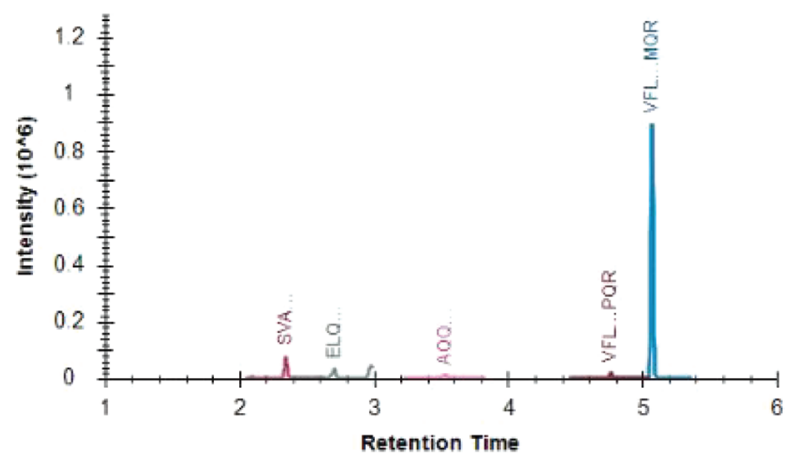

e) Soybeans peptides in frozen pasta

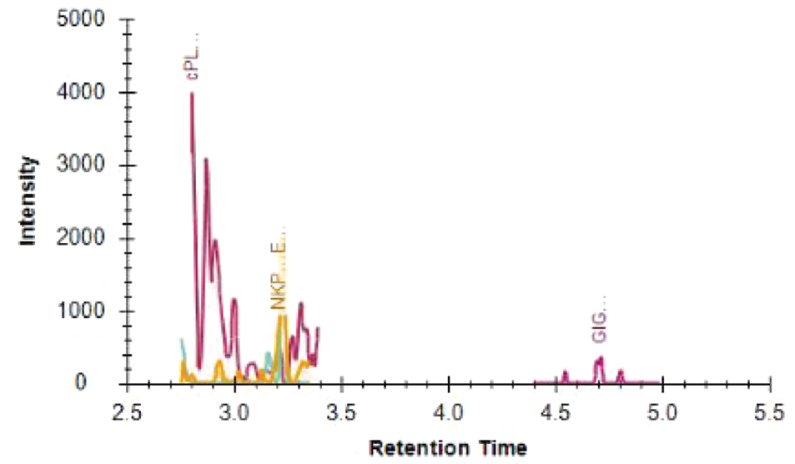

b) Shrimp peptides in whiteleg shrimp

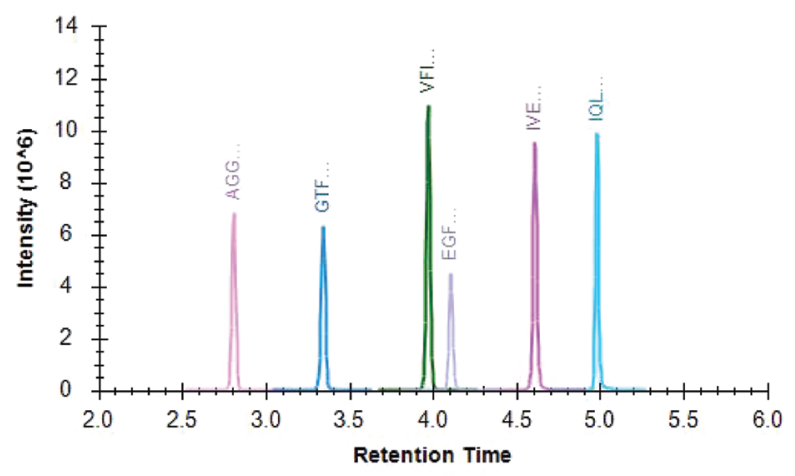

d) Wheat peptides in common wheat

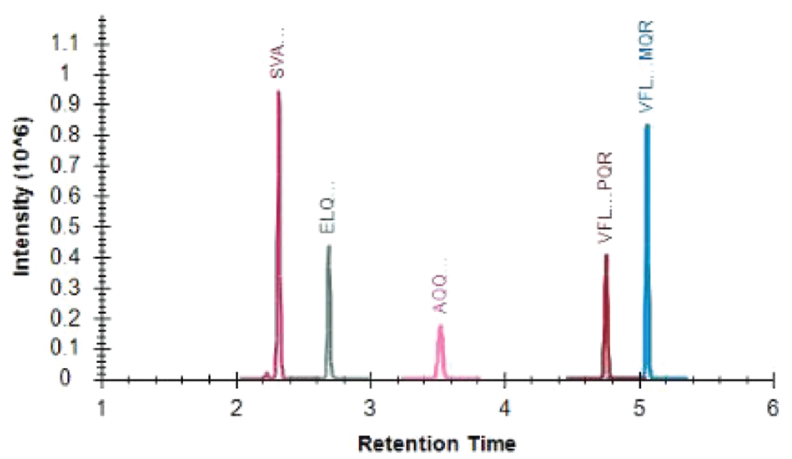

f) Soybeans peptides in soybeans

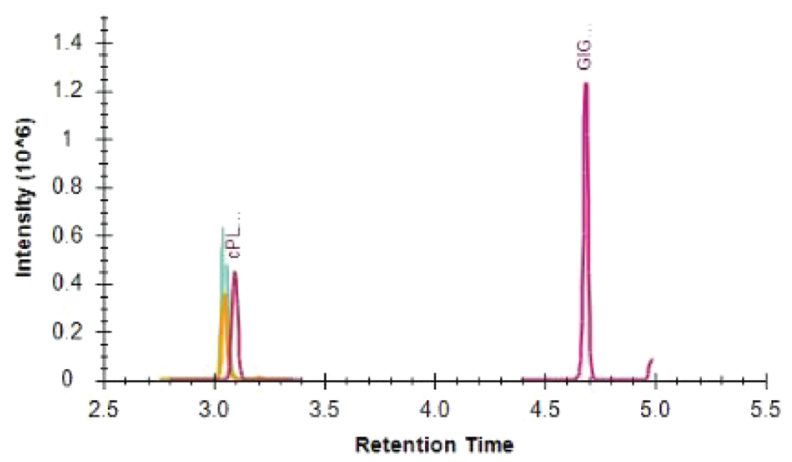

Figure 5. Comparison of chromatograms of each ingredients in pasta and row materials: (a) shrimp peptides in frozen pasta, (b) shrimp peptides in whiteleg shrimp, (c) wheat peptides in frozen pasta, (d) wheat peptides in common wheat, (e) soybeans peptides in frozen pasta, and (f) soybeans peptides in soybeans.

set to monitor 50 peptides selected from 21 proteins. We successfully detected multiple allergens from raw materials and thermally processed foods. We also demonstrated the method's ability to quantitate wheat peptides ranging from 3.9 to $250 \mathrm{ppm}(\mathrm{mg} / \mathrm{kg})$ using NIST SRM $1567 \mathrm{~b}$ wheat flour. These results confirm that this developed method could be used for monitoring of the 13 allergenic food ingredients from both raw material and thermally processed food.

\section{References}

(1) Gupta, R.S., Springston, E.E., Warrier, M.R., Smith, B., Kumar, R., Pongracic, J., \& Holl, J.L. (2011) Pediatrics 128, e9-e17. doi:10.1542/peds.2011-0204
(2) Kaminski, N.E., Kaplan, B.L.F., \& Holsapple, M.P. (2008) in Casarett \& Doull's Toxicology: The Basic Science of Poisons, 7th Ed., C.D. Klaassen (Ed.), McGraw-Hill Medical, New York, NY, pp 485-556

(3) Sicherer, S.H., \& Sampson, H.A. (2018) J. Allergy Clin. Immunol. 141, 41-58. doi:10.1016/j.jaci.2017.11.003

(4) Savage, J., \& Johns, C.B. (2015) Immunol. Allergy Clin. North Am. 35, 45-59. doi:10.1016/j.iac.2014.09.004

(5) Public Law 108-282, Title II (2004) Food Allergen Labeling and Consumer Protection Act, U.S. Food and Drug Administration, Silver Spring, MD

(6) U.S. Department of Agriculture, Food Safety and Inspection Service, Recall Summaries, https://www.fsis.usda.gov/wps/ portal/fsis/topics/recalls-and-public-health-alerts/recallsummaries (accessed October 25, 2018) 
(7) Gomaa, A., \& Ribereau, S., \& Boye, J. (2012) J. Nutr. Food Sci. S9. doi:10.4172/2155-9600.S9-001

(8) Khuda, S., Slate, A., Pereira, M., Al-Taher, F., Jackson, L., Diaz-Amigo, C., Bigley, C.E., Whitaker, T., \& Williams, M.K. (2012) J. Agric. Food Chem. 60, 4195-4203. doi:10.1021/ jf3001839

(9) Török, K., Hajas, L., Bugyi, Z., Balázs, G., \& Tömösközi, S. (2015) Acta Aliment. 44, 390-399. doi:10.1556/AAlim.2014.0018

(10) Sayers, R.L., Johnson, P.E., Marsh, J.T., Barran, P., Brown, H., \& Mills, E.N.C. (2016) Analyst 141, 4130-4141. doi:10.1039/C6AN00359A

(11) Röst, H.L., Rosenberger, G., Navarro, P., Gillet, L., Miladinović, S.M., Schubert, O.T., Wolski, W., Collins, B.C., Malmström, J., Malmström, L., \& Aebersold, R. (2014) Nat. Biotechnol. 32, 219-223. doi:10.1038/nbt.2841

(12) Gillet, L.C., Navarro, P., Tate, S., Röst, H., Selevsek, N., Reiter, L., Bonner, R., \& Aebersold, R. (2012)
Mol. Cell. Proteomics 11, O111.016717. doi:10.1074/mcp. O111.016717

(13) Uvackova, L., Skultety, L., Bekesova, S., McClain, S., \& Hajduch, M. (2013) J. Proteome Res. 12, 4862-4869. doi:10.1021/pr400336f

(14) Downs, M.L., \& Johnson, P. (2018) J. AOAC Int. 101, 146-151. doi:10.5740/jaoacint.17-0404

(15) Maclean, B., Tomazela, D.M., Shulman, N., Chambers, M., Finney, G.L., Frewen, B., Kern, R., Tabb, D.L., Liebler, D.C., \& Maccoss, M.J. (2010) Bioinformatics 26, 966-968. doi:10.1093/bioinformatics/btq054

(16) Rasheed, A., Xia, X., Yan, Y., Appels, R., Mahmood, T., \& He, Z. (2014) J. Cereal Sci. 60, 11-24. doi:10.1016/j. jcs.2014.01.020

(17) Baar, A., Pahr, S., Constantin, C., Scheiblhofer, S., Thalhamer, J., Giavi, S., Papadopoulos, N.G., Ebner, C., Mari, A., Vrtala, S., \& Valenta, R. (2012) J. Immunol. 189, 3018-3025. doi:10.4049/jimmunol.1200438 